\title{
Cancer-why do they make it so scary?
}

\section{Opinion}

There are a wide variety of cancers. AND, there are a wide variety of remedies...some with good research behind them and others not.

Let's understand cancer cells first. They have a different type of DNA. The DNA genetic program that makes sure that cells die is called apoptosis. That program is turned off in cancer cells. A second factor that is different in the cancer cells is the membrane. The electrophysiological membrane is depolarized which supports the maintenance of cancer stem cells. Further the mechanisms by which the membrane potentiation regulates cell proliferation, migration, and differentiation is different.

Further, normal cells take in a lot of oxygen and significantly less sugar. But cancer cells take in huge amounts of sugar and little oxygen. Cancer cells require about $4 \mathrm{x}$ as much sugar to make fuel compared to normal cells.

Tumors are also well equipped to increase angiogenesis (new blood vessel development). They want additional blood sources to take the nutrient they require to multiply and spread. Cancer research is slow because of a number of different issues:

a. The variety of different types of cancer cells

b. The variety of different body profiles that support cancers

c. Cancer cell membranes have ways to avoid our immune cell detection

d. We produce cancer cells all day every day - we need to strengthen our immune system

e. Dr. Bailer claims that "The five year survival statistics of the American Cancer Society are very misleading. They now count things that are not cancer, and, because we are able to diagnose at an earlier stage of the disease, patients falsely appear to live longer. Our whole cancer research in the past 20 years has been a total failure..."

f. Dr Robert Sharpe claims that " . . in our culture treating disease is enormously profitable, preventing it is not...Preventing the disease benefits no one except the patient."
Volume II Issue 2 - 2018

Holly Fourchalk

Choices unlimited for Health \& Wellness Ltd, Canada

Correspondence: Holly Fourchalk, Choices Unlimited for health and wellness, I475I 34a Ave, Surrey, BCV4P 0B3, Canada, Tel: +I604 7645 203, Email holly@choicesunlimited.ca

Received: October 26, 2017 | Published: April 20, 2018

g. Cancer societies spend more money on fundraising than on research

h. Research is too often based on old out dated theories

i. Research is designed to promote more funds - funds typically come from pharmaceutical companies - pharmaceutical companies don't want cures.

\section{Despite all that, we do know that}

a. Various herbs and foods can alter the membrane of cancer cells so they take in oxygen rather than sugar, thus making the insides of the cancer cells alkaline rather than acidic.

b. Various herbs and foods can turn apoptosis back on at the DNA genetic level of cancer cells, so that cancer cells actually die rather than keep multiplying.

c. Various herbs and foods can block the development of new blood vessels for tumors so that they can't access the nutrients that they require so what can we do with this information. People need to learn what their options are. Not be cornered or scared by conventional medicine.

Just like with conventional synthetic medication, real medicine has to be able to identify what kind of cancer and how and why it is there. Why are the cancer cells taking hold in this particular body. How do we feed the body without feeding the cancer cells. There are many ways to fight cancer in ways that are supportive to our body rather than destructive. Again, we need to help the consumer know and understand their options. 